\title{
Clinical features and outcome of MIS-C patients: an experience from Central Anatolia
}

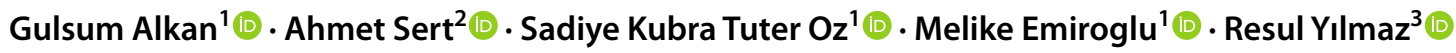

Received: 26 March 2021 / Revised: 22 April 2021 / Accepted: 25 April 2021 / Published online: 6 May 2021

(c) International League of Associations for Rheumatology (ILAR) 2021

\begin{abstract}
Background Multisystem inflammatory syndrome in children (MIS-C) is a new clinical condition characterized by signs of inflammation and multiorgan dysfunction due to cytokine storm associated with SARS-CoV-2. The clinical spectrum of MIS-C ranges from mild to severe, and even to mortal multisystem involvement. To guide clinicians, we evaluated detailed demographic characteristics, clinical features, laboratory findings, and outcomes of MIS-C cases.

Methods We performed a retrospective study of patients with MIS-C who were managed in the Department of Pediatric Infectious Disease in the Selcuk University Faculty of Medicine, Konya, Turkey. MIS-C patients were divided into three clinical severity groups (mild, moderate, and severe) and separated into three age groups ( $<5$ years, 5-10 years, $>10$ years). We compared the characteristics of MIS-C cases according to the severity of the disease and by age groups.

Result Thirty-six children with MIS-C were evaluated (52.8\% male, median age of 7.8 years). A clinical spectrum overlapping with Kawasaki disease (KD) was the most common presentation $(69.4 \%)$ in all age groups. The most common clinical symptoms were fever (100\%), mucocutaneous rash (69.4\%), and gastrointestinal symptoms (66.6\%). There was no statistically significant difference in echocardiographic abnormality between KD-like and the other clinical spectra $(p>0.05)$. All life-threatening rhythm disturbances were observed in severe cases. No patients died.

Conclusion It is important to increase the awareness of physicians about the MIS-C disease, which can present with different combinations of different systemic findings, so that patients can be diagnosed and treated in a timely manner.

\section{Key Points}

- A single tertiary centre study shows that children with MIS-C can present with different clinic spectra other than Kawasaki diseases.

- Electrocardiographic and echocardiographic evaluation is important in early diagnosis of children with MIS-C.

- Pro-BNP can be used as a screening test in the emergency room for children with prolonged and unexplained fever for determine early cardiac involvement of MIS-C.

- The lack of require biological agents and favourable outcomes in children with MIS-C may be related with administration of steroid therapy with IVIG in early stage of disease.
\end{abstract}

Keywords Cardiac involvement $\cdot$ Multisystem inflammatory syndrome in children $\cdot$ Outcomes $\cdot$ Symptoms $\cdot$ Treatment

Article summary The blockage of cytokine storm with intravenous immunoglobulin and steroid treatment in early stage of disease is a promising efficacy and safety treatment approach in MIS-C patients.

Gulsum Alkan

galkan-85@hotmail.com

Ahmet Sert

ahmetsert2@hotmail.com

Sadiye Kubra Tuter Oz

sadiyettr@hotmail.com

Melike Emiroglu

mkeser17@gmail.com

Resul Y1lmaz

drresul@gmail.com
1 Department of Pediatric Infectious Diseases, Selcuk University Faculty of Medicine, Konya, Turkey

2 Department of Pediatric Cardiology, Selcuk University Faculty of Medicine, Konya, Turkey

3 Department of Pediatric Critical Care, Selcuk University Faculty of Medicine, Konya, Turkey 


\section{Introduction}

Cases of coronavirus disease 2019 (COVID-19) among children have been less severe than those in adults. However, since late April 2020, multiple American and European institutions have reported paediatric cases with clinical similarities to toxic shock syndrome, Kawasaki disease (KD), or severe sepsis. This new life-threatening condition has been defined as multisystem inflammatory syndrome in children (MIS-C), which is related to severe acute respiratory syndrome coronavirus 2 (SARS-CoV-2) [1]. The mortality and morbidity in patients with MIS-C are vastly different from the benign course of COVID-19 in children [2]. The majority of patients have either known family exposures or serologic evidence of SARS-CoV-2, specifically positive $\operatorname{IgG}$ and negative $\operatorname{IgM}$, supporting the thesis that MIS-C represents an immune-mediated complication rather than an acute infection [2]. The underlying pathological mechanisms of MIS-C are considered a dysregulated inflammatory response, possibly triggered by SARS-CoV-2, rather than direct viral toxicity. A cytokine storm can result from ability of SARS-CoV-2 to block types I and III interferon responses [3, 4].

Although MIS-C is a multisystemic disease, cardiovascular manifestations are most prominent [5]. Other common clinical findings of MIS-C are persistent fever, gastrointestinal (vomiting, diarrhoea, abdominal pain) symptoms, mucocutaneous rash reminiscent of $\mathrm{KD}$, and (rarely) respiratory symptoms. MIS-C cases present with a wide clinical spectrum, overlapping with the KD spectrum (persistent fever, polymorphic rash, mucosal changes, and conjunctivitis) or with severe acute COVID19. Notably, other subset of the group has more frequent gastrointestinal symptoms and cardiovascular involvement. Although some clinical features are similar, MIS-C cases differ from KD, especially as cardiac dysfunction and hypotension are more commonly seen in MIS-C [3]. Most of MIS-C cases with respiratory symptoms, such as cough, shortness of breath, pneumonia, and acute respiratory distress syndrome, have a high mortality rate and positive SARS-CoV-2 polymerase chain reaction (PCR) without seropositivity [6, 7].

Because MIS-C is described as a life-threatening multisystem condition, treatment options are needed urgently. The aim of MIS-C treatment is to supress systemic inflammation, improve cardiac function, and prevent long-term sequelae. Current treatment regimens derived from experience with KD and adult experience with COVID-19 consist of cardiac support, immunomodulation, and anticoagulation therapy [2]. As the experience of clinicians with long-term outcomes of MIS-C cases increases, treatment approaches will become clearer. We retrospectively investigated demographic, epidemiological, clinical, laboratory features, cardiac involvement, and treatment modalities of MIS-C cases, in order to compile and disseminate information urgently needed at present by the medical community.

\section{Methods}

We reviewed the medical records of 36 patients treated with MIS-C between September 2020 and February 2021 at the Department of Pediatric Infectious Disease in the Selcuk University Faculty of Medicine, Konya, Turkey. The study protocols were approved by our hospital's ethics committee (approval number: 2021/58). Patients were included for study based on the terms of the case definition by the Centres for Disease Control and Prevention (CDC): Characteristics principles for diagnosis as follows:

1. Age $<21$ years

2. Clinical presentation consistent with MIS-C, including all of the following:

- Fever:

- Documented fever $>38,0{ }^{\circ} \mathrm{C}\left(100,4{ }^{\circ} \mathrm{F}\right)$ for $>24$ hours or report of subjective fever lasting $>24$ hours

- Laboratory evidence of inflammation, including any of the following:

- Elevated C-reactive protein (CRP), erythrocyte sedimentation rate (ESR), fibrinogen, procalcitonin, D-dimer, ferritin, LDH, interleukin 6 (IL-6) level

- Neutrophilia, lymphocytopeniae, hypoalbunemia

- Multisystem ( $\geq 2)$ organ involvement

- Cardiovascular (eg, shock, elevated troponin, elevated brain-type natriuretic peptide , abnormal echocardiography, arrhythmia)

- Respiratory (eg, pneumonia, ARDS, pulmonary embolism)

- Renal (renal failure)

- Neurologic (eg, seizure, stroke, aseptic meningitis)

- Hematologic (eg, coagulopathy)

- Gastrointestinal (eg, abdominal pain, vomiting, diarrhoea, elevated liver enzymes, ileus)

- Severe illness requiring hospitalization

3. No alternative plausible diagnosis 
4. Evidence of infection with severe acute respiratory syndrome coronavirus 2 (SARS-CoV-2)

Any of the following

- Positive SARS-CoV-2 reverse-transcriptase polymerase chain reaction (RT-PCR)

- Positive serology

- COVID-19 exposure to persons in the past month [8].

Children were identified in three subgroups based of main clinical manifestation: MIS-C overlapping with KD; overlapping with severe acute COVID-19; no overlap with acute COVID-19 or KD (mainly cardiovascular and gastrointestinal involvement). Clinical severity was defined as follows: mild MIS-C with no vasoactive requirement or respiratory support and minimal organ injury; moderate MIS-C with mild or isolated organ injury; severe MIS-C with moderate or severe organ injury, including moderate to severe ventricular dysfunction [9]. Children were also separated into three age groups ( $<5$ years, $5-10$ years, $>10$ years). We aimed to define the clinical spectrum of MIS-C patients by age groups and compare with KD which is mostly seen under 5 years of age.

Patients were evaluated for demographic characteristics, history of past COVID-19, history of contact with confirmed or suspected cases of COVID-19, presenting symptoms, clinical and laboratory features, radiological findings, cardiac involvement, treatment modalities, and outcomes. Laboratory data on admission and during hospitalisation were also collected, including complete blood count with differential, routine biochemical tests, inflammatory markers (CRP, ESR, procalcitonin, IL-6, ferritin), D-dimer, troponin, pro-brain-type natriuretic peptide (pro-BNP), international normalized ratio (INR), and activated partial thromboplastin time (aPTT). All cases underwent antibody testing and nasopharyngeal RT-PCR testing for SARS-CoV-2. An enzymelinked immunosorbent assay (ELISA) was used to identify serum anti-SARS-CoV-2 IgM and IgG (upper limit: 2 ng/ $\mathrm{mL})$.

The echocardiography (ECHO) and ECG findings for the patients were recorded at the time of admission, and on the 7 th and 14th days. We retrospectively collected 12-lead ECGs from children presenting with MIS-C. All ECGs were evaluated by a single paediatric cardiologist. Myocarditis was defined as cardiac dysfunction (ventricular dysfunction or decreased ejection fraction) on ECHO with an elevated troponin level or pro-BNP. Hepatitis was defined as an elevation of ALT $>50 \mathrm{U} / \mathrm{L}$ and AST $>50 \mathrm{U} / \mathrm{L}$, in the absence of other etiologic factors.

The therapeutics included intravenous immunoglobulin (IVIG), glucocorticoids, enoxaparin, acetyl salicylic acid (ASA), and vasoactive drugs, and the length of hospitalisation was noted. The recovery times of cardiac functions, arrhythmias, and cardiac sequelae were recorded.

\section{Statistical analysis}

Statistical analysis was performed using a computer software package (SPSS for Windows, version 21.0). The Shapiro-Wilk test and Kolmogorov-Smirnov test were applied to check the distribution of parameters. Based on whether the data were normally distributed or not, data were analysed by parametric or nonparametric methods. If data were distributed normally, parameters were reported as mean \pm standard deviation; if not, they were presented as median with interquartile range (IQR). Categorical variables were presented as number and percentage. The chi-square test was used to compare categorical variables between groups. Relative risk (RR) and its $95 \%$ confidence interval (95\% CI) were calculated to compare outcomes between groups. The Kruskal-Wallis analysis of variance test was used to compare groups, and the Bonferroni-corrected Mann-Whitney $U$ test was used as a more conservative measure of significance for multiple comparisons. Results were considered significant if $p<0.05$ or, in case of $k$ comparisons, when $p<0.05 / k$.

\section{Results}

\section{Demographics and epidemiology}

A total number of 36 patients (19 males, 52.8\%) with the median age at diagnosis of 7.8 years (range: 17 months -17 years) were enrolled to study. All children were previously healthy, except one obese adolescent. There were no statistically significant differences between age and gender of patients regarding the severity of the disease and the clinical spectrum of diseases $(p>0.05)$. Although epidemiological history was positive in only $20(55.5 \%)$ patients, COVID-19 was confirmed in $91.6 \%$ of patients (Table 1). Eight patients reported COVID-19 symptoms before the onset of MIS-C, and the median interval was 28 days (range: 21-63 days).

\section{Symptoms and clinical spectrum}

The most common clinical form of MIS-C was the severe form $(47.2 \%)$, and the most common clinical phenotype was the KD phenotype (69.4\%) (Table 1). None of the patients met the criteria for the classic form of KD. Three of the patients presented with respiratory distress and bilateral diffuse infiltrates on chest X-rays, suggesting acute COVID-19. Eight of the patients presented only with gastrointestinal symptoms and had serology positivity and cardiac involvement by ECHO. There was no statistically significant 
Table 1 Demographics, clinical findings, echocardiographic, and electrocardiographic findings of patients with MIS-C

\begin{tabular}{|c|c|c|c|c|c|c|}
\hline & Total patients & Mild MIS-C & Moderate MIS-C & Severe MIS-C & $p$ values & Relative risk $(95 \% \mathrm{CI})$ \\
\hline Characteristics $(n, \%)$ & $36(100 \%)$ & $13(36.1 \%)$ & $6(16.7 \%)$ & $17(47.2 \%)$ & & \\
\hline Gender (Male/female) & $19 / 17(52.8 / 47.2)$ & $8 / 5(61.5 / 38.5)$ & $3 / 3(50 / 50)$ & $8 / 9(42.1 / 52.9)$ & 0.690 & $1.230(0.398-1.589)$ \\
\hline Age (month)* & $94.5(17-204)$ & $71(17-166)$ & $103.5(28-132)$ & $109(17-204)$ & 0.362 & \\
\hline \multicolumn{7}{|l|}{ SARS-CoV-2 infection evidence } \\
\hline Recent COVID-19 infection & $8(22.2 \%)$ & $4(30 \%)$ & $1(16.6 \%)$ & $3(17.6 \%)$ & 0.650 & $1.491(0.418-5.326)$ \\
\hline Known COVID-19 exposure & $12(33.3 \%)$ & $4(30.7 \%)$ & $2(33.3 \%)$ & $6(35.2 \%)$ & 0.581 & $0.805(0.434-1.495)$ \\
\hline SARS-CoV-2 PCR positivity & $1(2.7 \%)$ & - & - & $1(5.8 \%)$ & 0.563 & NA \\
\hline SARS-CoV-2 IgG positivity & $32(88.8 \%)$ & $12(92.3 \%)$ & $5(83.3 \%)$ & $15(88.2 \%)$ & 0.938 & $0.954(0.735-1.239)$ \\
\hline \multicolumn{7}{|l|}{ Kawasaki disease symptoms } \\
\hline Fever & $36(100 \%)$ & $13(100 \%)$ & $6(100 \%)$ & $17(100 \%)$ & NA & NA \\
\hline Rash & $21(58.3 \%)$ & $7(53.8 \%)$ & $1(16.6 \%)$ & $13(76.4 \%)$ & $0.035^{\ddagger}$ & $0.551(0.305-0.993)$ \\
\hline Conjunctivitis & $25(69.4 \%)$ & $8(61.5 \%)$ & $3(50 \%)$ & $14(82.3 \%)$ & 0.248 & $0.703(0.452-1.094)$ \\
\hline Periorbital oedema and erythema & $7(19.4 \%)$ & - & $1(16.6 \%)$ & $6(35.2 \%)$ & $0.018^{\dagger}$ & $0.149(0.020-1.117)$ \\
\hline Strawberry tongue & $21(58.3 \%)$ & $8(61.5 \%)$ & $3(50 \%)$ & $10(58.8 \%)$ & 0,89 & $0.703(0.452-1.094)$ \\
\hline Cervical lymphadenopathy $(>1.5 \mathrm{~cm})$ & $5(13.8 \%)$ & - & $1(16.6 \%)$ & $4(23.5 \%)$ & 0.178 & $0.179(0.023-1.383)$ \\
\hline Abdominal pain & $20(55.5 \%)$ & $8(61.5 \%)$ & $4(66.6 \%)$ & $8(47 \%)$ & 0.611 & $1.342(0.729-2.470)$ \\
\hline Vomiting & $12(33.3 \%)$ & $5(38.5 \%)$ & $2(33.3 \%)$ & $5(29.4 \%)$ & 0.873 & $1.253(0.488-3.216)$ \\
\hline Gastroenteritis & $10(27.7 \%)$ & $3(23 \%)$ & $3(50 \%)$ & $4(23.5 \%)$ & 0.412 & $1.342(0.454-3.963)$ \\
\hline Mesenteric lymphadenopathy & $5(13.8 \%)$ & $2(20 \%)$ & $1(16.6 \%)$ & $2(11.7 \%)$ & 1 & $1(0.202-4.955)$ \\
\hline Hepatitis & $9(25 \%)$ & $3(23 \%)$ & - & $6(35.2 \%)$ & 0.225 & $0.447(0.132-1.518)$ \\
\hline Prerenal insufficiency & $4(11.1 \%)$ & - & - & $4(23.5 \%)$ & $0.038^{\dagger}$ & $(1.042-1.925)$ \\
\hline Neurological sign & $14(38.8 \%)$ & $6(46.1 \%)$ & - & $8(47 \%)$ & 0.101 & $0.671(0.292-1.542)$ \\
\hline Haematological involvement & $34(94.4 \%)$ & $12(92.3 \%)$ & $5(83.3 \%)$ & $17(100 \%)$ & 0.283 & $(0.767-1.044)$ \\
\hline Thrombocytopenia & $10(27.4 \%)$ & $2(15.3 \%)$ & $1(16.6 \%)$ & $7(41.1 \%)$ & 0.236 & $0.383(0.117-1.253)$ \\
\hline Lymphopenia & $12(33.3 \%)$ & $2(15.3 \%)$ & - & $10(58.8 \%)$ & $0.03^{\dagger+}$ & $0.179(0.045-0.704)$ \\
\hline D-dimer abnormality & $32(88.8 \%)$ & $11(84.6 \%)$ & $4(66.6 \%)$ & $17(100 \%)$ & $0.043^{\ddagger}$ & $(0.626-0.996)$ \\
\hline Coagulation abnormalities & $10(27.7 \%)$ & $3(23 \%)$ & $2(33.3 \%)$ & $5(29.4 \%)$ & 0.929 & $0.944(0.331-2.692)$ \\
\hline ECG abnormality & $17(47.2 \%)$ & $1(15.3 \%)$ & $4(83.3 \%)$ & $12(64.7 \%)$ & $0.002^{\dagger ¥}$ & $0.373(0.165-0.840)$ \\
\hline Sinus tachycardia & $8(22.2 \%)$ & $1(7.6 \%)$ & $3(50 \%)$ & $4(23.5 \%)$ & 0.117 & $0.895(0.264-3.036)$ \\
\hline Incomplete right bundle branch block & $5(13.8 \%)$ & $1(7.6 \%)$ & $1(16.6 \%)$ & $3(17.6 \%)$ & 0.720 & $0.596(0.113-3.154)$ \\
\hline First-degree AV block & $4(11.1 \%)$ & - & $1(16.6 \%)$ & $3(17.6 \%)$ & 0.280 & $0.298(0.034-2.603)$ \\
\hline ST elevation & $1(2.7 \%)$ & - & - & $1(5.8 \%)$ & 0.563 & $(0.943-1.197)$ \\
\hline Normal ECG rhythm & $19(52.7 \%)$ & $12(92.3 \%)$ & $2(33.3 \%)$ & $5(29.4 \%)$ & $0.002^{\dagger ¥}$ & $2.505(1.144-5.487)$ \\
\hline ECHO findings & $30(83.3 \%)$ & $8(61.5 \%)$ & $6(100 \%)$ & $16(94.1 \%)$ & $0.029^{\dagger}$ & $0.783(0.584-1.050)$ \\
\hline Valve insufficiency & $25(69.4 \%)$ & $7(53.8 \%)$ & $5(83.3 \%)$ & $13(76.4 \%)$ & 0.296 & $0.826(0.536-1.273)$ \\
\hline Left ventricular dysfunction & $11(30.5 \%)$ & - & - & $11(64.7 \%)$ & $0.00^{\dagger \dagger}$ & $(1.489-5.393)$ \\
\hline Right ventricular dysfunction & $3(8.3 \%)$ & - & - & $3(17.6 \%)$ & 0.176 & $(0.974-1.513)$ \\
\hline Ventricular dilatation with normal EF & $5(13.8 \%)$ & - & $2(33.3 \%)$ & $3(17.6 \%)$ & $0.013^{¥}$ & $1.342(0.254-7.096)$ \\
\hline Coronary dilatation & $4(11.1 \%)$ & - & - & $4(23.3 \%)$ & $0.038^{\dagger \neq}$ & $(1.005-1.702)$ \\
\hline Pericardial effusion & $3(8.3 \%)$ & $1(7.6 \%)$ & - & $2(11.7 \%)$ & 0.66 & $0.447(0.044-4.505)$ \\
\hline Clinical features overlapping with KD & $25(69.4 \%)$ & $11(84.6 \%)$ & $2(33.3 \%)$ & $12(70.5 \%)$ & 0.078 & $0.895(0.565-1.418)$ \\
\hline Clinical features overlapping with COVID-19 & $3(8.3 \%)$ & - & $1(16.6 \%)$ & $2(11.7 \%)$ & 0.370 & $0.447(0.044-4.505)$ \\
\hline Without overlapping with COVID-19 or KD & $8(22.2 \%)$ & $2(15.3 \%)$ & $3(50 \%)$ & $3(17.6 \%)$ & 0.198 & $1.491(0.418-5.326)$ \\
\hline Length of stay in hospital* & $8(4-18)$ & $6(4-18)$ & $7(5-10)$ & $10(7-17)$ & $0.01^{\dagger}$ & \\
\hline
\end{tabular}

Abbreviations: $A V$ block, atrioventricular node block; $E C G$, electrocardiography; $E C H O$, echocardiography; $E F$, ejection fraction; $I g G$, immunoglobulin G; KD, Kawasaki disease; $N A$, not applicable; $S A R S-C o V-2$, severe acute respiratory syndrome coronavirus $2 ; P C R$, polymerase chain reaction

Categorical variables were presented as number and percentage

*Values were presented as median with range

Relative risk (RR) was calculated between the severe MIS-C group and the combined mild-to-moderate MIS-C group, as the number of cases was relatively small

${ }^{\dagger} p<0.05$; severe MIS-C versus mild MIS-C

${ }^{\ddagger} p<0.05$; severe MIS-C versus moderate MIS-C

${ }^{¥} p<0.05$; moderate MIS-C versus mild MIS-C 
difference between the clinical phenotype and the severity of the disease $(p>0.05)$. Fever was detected in all patients on admission or during hospitalisation. Median duration of fever prior to admission was 3.5 days, and its median value was $39^{\circ} \mathrm{C}$ (range: $38-40{ }^{\circ} \mathrm{C}$ ). MIS-C cases with myocarditis had statistically significant longer fever duration $(p=0.049)$ and high-grade fever $(p=0.026)$ before admission. The other common presentations of patients were mucocutaneous symptoms (69.4\%) including conjunctivitis and rash; gastrointestinal symptoms $(66.6 \%)$ including vomiting, abdominal pain and/or diarrhoea; and neurologic findings $(38.8 \%)$ including headache, irritability, aseptic meningitis, or periorbital oedema-erythema (19.4\%). Myocarditis $(52.7 \%)$ was detected in all age groups and all clinical phenotypes of MIS-C $(p>0.05)$. One patient who presented with KD features also had epididymo-orchitis. There was no statistically significant difference between neurological and gastrointestinal symptoms and the severity of disease $(p>0.05)$. Periorbital oedema-erythema $(p=0.018)$ and rash $(p=0.03)$ were significantly more common in severe disease $(p<0.05)$. Conjunctivitis was detected more frequently in the ages of 5 to 10 years than in $<5$ years of age $(p=0.036)$. Symptoms and the clinical spectrum of MIS-C cases according to age and clinical severity are shown in Tables 1 and 3.

\section{Laboratory findings and radiological imaging}

Laboratory parameters were evaluated according to the clinical severity of disease as summarized in Table 2. Hematologic abnormalities detected were neutrophilia (33.3\%), neutropenia $(2.7 \%)$, lymphopenia $(33.3 \%)$, thrombocytopenia $(27.7 \%)$, and increased D-dimer $(88.8 \%)$. INR levels were high in 10 patients (range: $1.3-2$ ). Inflammation markers were elevated as CRP (97.2\%), IL-6 (86.1\%), ESR (75\%), procalcitonin $(75 \%)$, and ferritin $(44.4 \%)$. Elevated troponin and pro-BNP were detected in $33.3 \%$ and $72.2 \%$ of the patients, respectively. While hypoalbuminemia (47.2\%) and hyponatremia (55.5\%) were seen in all clinical spectra of disease, high-level urea (11.1\%) was detected only in patients with severe MIS-C $(p=0.038)$. Lymphopenia, high levels of procalcitonin, IL-6, ferritin, D-dimer, troponin, proBNP, ALT, and urea were related to the severity of MIS-C $(p<0.05)$.

The median anti-SARS-CoV-2 IgG level was $32.17 \mathrm{ng} /$ $\mathrm{mL}$ (range: 5-208). There was no statistically significant relationship between the severity of the disease and the $\operatorname{IgG}$ levels $(p=0.28)$.

All patients were evaluated with chest radiography regardless of symptoms to detect end organ damage. Eight patients underwent thorax computed tomography (CT) to evaluate abnormalities shown on chest radiography. Three of them had ground-glass opacities and local patchy shadowing, and two had pleural effusion. One week after the treatment was initiated, chest radiography markedly improved, and after 2 weeks, CT results returned to normal. Abdominal ultrasound (US) was performed in patients with abdominal pain and/or elevated liver enzymes. Abdominal US examination of 22 patients, mesenteric lymphadenitis ( $n=5$, mean $2 \times 1 \mathrm{~cm}$ diameter), ascites ( $n=4$, mean $4 \mathrm{~cm}$ thickness), one from each of the ileitis, cholecystitis, and inguinal lymphadenitis were detected. After 2 weeks, ascites and lymphadenitis disappeared.

\section{Cardiac testing}

Abnormal ECHO (83.3\%), abnormal ECG (47.2\%), elevated pro-BNP $(72.2 \%)$, and/or elevated troponin $(33.3 \%)$ were detected on admission. The frequency of ECHO abnormality by age groups ( $<5$ years, $5-10$ years, $>10$ years) was $88.8 \%$, $85.7 \%$, and $76.9 \%$, respectively. Cardiac involvement was more frequent in children older than 10 years of age than in those $<5$ years of age by ECHO $(p<0.05)$. The most common ECHO findings were valve insufficiencies $(69.4 \%)$ and left ventricular dysfunction (30.5\%). While mild mitral valve insufficiency was detected in $69.4 \%$ of patients, the proportion of tricuspid, aortic, and pulmonary valve insufficiency was $19.4 \%, 13.8 \%$, and $2.7 \%$, respectively. More than 1 valve insufficiency was detected in 10 patients with severe MIS-C. Valve insufficiencies were seen in all clinic severity types of MIS-C, while ventricular dysfunction and coronary dilatation were seen in only severe cases (Table 1).

There was no significant difference in ECHO abnormality between clinical spectra of MIS-C $(p>0.05)$. Dilatation of the coronary arteries ( $\mathrm{Z}$ score $2.5-3)$ was found in four patients $(11.1 \%)$, including one patient (5-10 years) with dilatation of the proximal left main and three patients ( $>10$ years) with dilatation of the right coronary artery. Three of these cases presented with KD features, and one of them presented only GI symptoms. These patients had prolonged fever ( $>5$ days) and high-grade fever $\left(>39^{\circ} \mathrm{C}\right)$ before hospital admission.

The most common ECG abnormalities were sinus tachycardia (22.2\%), followed by incomplete right bundle branch block (RBBB) and first-degree AV block on admission. During hospitalisation, sinus arrhythmia was detected in $33.3 \%$ of the patients. Non-sustained uniform ventricular tachycardia temporarily occurred in two patients with severe myocarditis. ECG abnormality was more common in $5-10$ years $(50 \%)$ and $>10$ years $(69.2 \%)$ than $<5$ years. Normal ECG rhythm was detected in $92.3 \%$ of mild cases. There was no significant difference in ECG rhythm disorders between the clinical spectra of MIS-C $(p>0.05)$. The median troponin and pro-BNP measurement in serum was $9.4 \mathrm{ng} / \mathrm{L}, 2.539 \mathrm{pg} / \mathrm{mL}$. In the first evaluation, pro-BNP levels were found to be high in eight patients who presented with only gastrointestinal symptoms. While median levels of 
Table 2 Laboratory findings of the patients with MIS-C

\begin{tabular}{|c|c|c|c|c|c|c|}
\hline & Total patient & Mild MIS-C (1) & Moderate MIS-C (2) & Severe MIS-C (3) & $p$ value $^{¥}$ & $p$ value $^{\epsilon}$ \\
\hline Variable & $n=36(100 \%)$ & $n=13(36.1 \%)$ & $n=6(16.7 \%)$ & $n=17(47.2 \%)$ & & \\
\hline $\mathrm{WBC}(\mathrm{K} / \mu \mathrm{L})$ & $10,700 \pm 4842$ & $11,162 \pm 6092$ & $12,583 \pm 4495$ & $9682 \pm 3813$ & 0.373 & \\
\hline Neutrophil count $(\mathrm{K} / \mu \mathrm{L})^{*}$ & $7650(6200)$ & $7400(7150)$ & $10,450(7400)$ & $7500(5450)$ & 0.628 & \\
\hline Lymphocyte count $(\mathrm{K} / \mu \mathrm{L})^{*}$ & $1650(1575)$ & $1900(1100)$ & $2000(1175)$ & $1000(1450)$ & 0.026 & $\begin{array}{l}1 \text { vs. } 2: 0.69 \\
1 \text { vs. } 3: 0.032 \\
2 \text { vs. } 3: \mathbf{0 . 0 2 3}\end{array}$ \\
\hline Platelet count $(\mathrm{K} / \mu \mathrm{L})$ & $199,190 \pm 86,355$ & $217,310 \pm 82,820$ & $286,670 \pm 110,176$ & $154,470 \pm 4597$ & 0.005 & $\begin{array}{l}1 \text { vs. } 2: 0.114 \\
1 \text { vs. } 3: 0.016 \\
2 \text { vs. } 3: 0.009\end{array}$ \\
\hline $\operatorname{ESR}(>20 \mathrm{~mm} / \mathrm{h})^{*}$ & $49(56)$ & $42(60)$ & $66(53)$ & $51(45)$ & 0.568 & \\
\hline C-reactive protein $(>8 \mathrm{mg} / \mathrm{L})^{*}$ & $148(158)$ & $91.5(153)$ & $71.6(230)$ & $195(165)$ & 0.168 & \\
\hline Procalcitonin $(>0.5 \mathrm{ng} / \mathrm{L})^{*}$ & $1.6(8.2)$ & $1.5(2.3)$ & $0.3(1.4)$ & $4.8(27)$ & 0.001 & $\begin{array}{l}1 \text { vs. } 2: 0.188 \\
1 \text { vs. } 3: 0.006 \\
2 \text { vs. } 3: \mathbf{0 . 0 0 3}\end{array}$ \\
\hline $\mathrm{IL}-6(>6.4 \mathrm{pg} / \mathrm{mL})^{*}$ & 76. (132) & 47 (113) & $23.7(67)$ & $146(214)$ & 0.006 & $\begin{array}{l}1 \text { vs. } 2: 0.161 \\
1 \text { vs. } 3: \mathbf{0 . 0 4 2} \\
2 \text { vs. } 3: \mathbf{0 . 0 0 3}\end{array}$ \\
\hline Troponin $(>17.5 \mathrm{ng} / \mathrm{L}) *$ & $9.4(28)$ & $6.6(12)$ & $6.65(94)$ & $25.6(40)$ & 0.047 & $\begin{array}{l}1 \text { vs. } 2: 0.9 \\
1 \text { vs. } 3: 0.022 \\
2 \text { vs. } 3: 0.09\end{array}$ \\
\hline Pro-BNP $(>125 \mathrm{pg} / \mathrm{mL}) *$ & $2539(7125)$ & $1500(2230)$ & $543(1691)$ & $7834(17,932)$ & 0.003 & $\begin{array}{l}1 \text { vs. } 2: 0.216 \\
1 \text { vs. } 3: 0.008 \\
2 \text { vs. } 3: \mathbf{0 . 0 0 5}\end{array}$ \\
\hline $\mathrm{LDH}(\mathrm{U} / \mathrm{L})^{*}$ & $312(100)$ & $284(129)$ & $281(70)$ & $352(101)$ & 0.118 & \\
\hline $\operatorname{INR}(>1.2)$ & $1.20 \pm 0.23$ & $1.27 \pm 0.358$ & $1.19 \pm 0.153$ & $1.15 \pm 0.14$ & 0.836 & \\
\hline D-dimer $(>500 \mathrm{ng} / \mathrm{mL})^{*}$ & $2270(2617)$ & $2061(2278)$ & $764(1520)$ & $3240(2504)$ & 0.01 & $\begin{array}{l}1 \text { vs. } 2: 0.096 \\
1 \text { vs. } 3: 0.052 \\
2 \text { vs. } 3: 0.008\end{array}$ \\
\hline Ferritin $(>336 \mathrm{ng} / \mathrm{mL}) *$ & $285(519)$ & $258(313)$ & $82.20(102)$ & $601(517)$ & $<0.0001$ & $\begin{array}{l}1 \text { vs. } 2: 0.079 \\
1 \text { vs. } 3: 0.002 \\
2 \text { vs. } 3: \mathbf{0 . 0 0 1}\end{array}$ \\
\hline $\operatorname{AST}(>50 \mathrm{U} / \mathrm{L})^{*}$ & $33.5(23)$ & $30(25)$ & $26.5(18)$ & $35(40)$ & 0.241 & \\
\hline $\operatorname{ALT}(>50 \mathrm{U} / \mathrm{L}) *$ & $26(24)$ & $15(16.5)$ & $26.5(14)$ & $34(38)$ & $\mathbf{0 . 0 3 0}$ & $\begin{array}{l}1 \text { vs. } 2: 0.792 \\
1 \text { vs. } 3: \mathbf{0 . 0 3 1} \\
2 \text { vs. } 3: \mathbf{0 . 0 3}\end{array}$ \\
\hline Albumin $(<3.5 \mathrm{~g} / \mathrm{dL})$ & $3.47 \pm 0.65$ & $3.66 \pm 0.63$ & $3.71 \pm 0.49$ & $3.24 \pm 0.66$ & 0.097 & \\
\hline Sodium $(<135 \mathrm{mEq} / \mathrm{L})$ & $134 \pm 4$ & $135.3 \pm 3.3$ & $135.8 \pm 2.92$ & $132.4 \pm 4.51$ & 0.162 & \\
\hline Urea $(\mathrm{mg} / \mathrm{dL})^{*}$ & $21(11)$ & $19(12.5)$ & $20(7)$ & $25(16)$ & 0.173 & \\
\hline Creatinine (mg/dL) & $0.498 \pm 0.211$ & $0.412 \pm 0.14$ & $0.455 \pm 0.149$ & $0.578 \pm 0.24$ & 0.103 & \\
\hline SARS-CoV-2 IgG level* & $32.17(5-208)$ & $30.08(6-69)$ & $29.20(5-45)$ & $43(7-208)$ & 0.28 & \\
\hline
\end{tabular}

Categorical variables were presented as number and percentage. If data were distributed normally, parameters were reported as mean \pm standard deviation; if not, they were presented as median with IQR. Results were compared using the Kruskal-Wallis test followed by the Bonferroni-corrected Mann-Whitney $U$ test. Significance was determined by $p<0.05$ for the Kruskal-Wallis test and $p<0.016(p=0.05 / 3)$ for the Bonferroni correction. Statistically significant differences are highlighted in bold

${ }^{¥}$ Kruskal-Wallis test, ${ }^{€}$ Bonferroni-corrected Mann-Whitney $U$ test

pro-BNP were $2.112 \mathrm{pg} / \mathrm{mL}$, median levels of troponin levels were $13.5 \mathrm{ng} / \mathrm{L}$ in these patients. Although there was no significant difference in levels of cardiac enzymes according to clinical spectra, higher levels of troponin $(p=0.047)$ and pro-BNP ( $p=0.003)$ were observed in severe MIS-C cases. ECHO, ECG findings, troponin, and pro-BNP level results by age and clinical severity are summarized in Tables 1, 2, and 3 .

\section{Treatment and outcomes}

On admission, $30.5 \%$ of the patients presented with cardiogenic shock. They received positive inotropic therapy for a median on 5 days (range: $2-8$ ). Four (11.1\%) patients were followed in the paediatric intensive care unit, and the median length of stay was 4 days (range: 1-14). None of these patients needed mechanical ventilation or extracorporeal 
Table 3 Clinical manifestations of the patients with MIS-C according to age groups

\begin{tabular}{|c|c|c|c|c|c|}
\hline Clinical Manifestations & $\begin{array}{l}\text { Total Patient } \\
\mathrm{n}=36(100 \%)\end{array}$ & $\begin{array}{l}<5 \text { years } \\
\mathrm{n}=9(25 \%)\end{array}$ & $\begin{array}{l}5-10 \text { years } \\
n=14(38.8 \%)\end{array}$ & $\begin{array}{l}>10 \text { years } \\
n=13(36.1 \%)\end{array}$ & $\begin{array}{l}\mathrm{P} \text { value } \\
\mathrm{X}^{2}=0.05\end{array}$ \\
\hline \multicolumn{6}{|l|}{ Severity of MIS-C } \\
\hline Mild & $13(36.1 \%)$ & $4(44.4 \%)$ & $5(35.7 \%)$ & $4(30.7 \%)$ & 0.805 \\
\hline Moderate & $6(16.6 \%)$ & $2(22.2 \%)$ & $3(21.4 \%)$ & $1(7.6 \%)$ & 0.554 \\
\hline Severe & $17(47.2 \%)$ & $3(33.3 \%)$ & $6(42.8 \%)$ & $8(61.5 \%)$ & 0.392 \\
\hline \multicolumn{6}{|l|}{ Clinical appearance } \\
\hline Overlapping with KD & $25(69.4 \%)$ & $6(66.6 \%)$ & $12(85.7 \%)$ & $7(53.8 \%)$ & 0.396 \\
\hline Overlapping with acute COVID-19 & $3(8.3 \%)$ & - & $1(7.1 \%)$ & $2(15.3 \%)$ & 0.429 \\
\hline $\begin{array}{l}\text { Without overlapping with acute COVID- } 19 \\
\text { or KD }\end{array}$ & $8(22.2 \%)$ & $3(33.3 \%)$ & $1(7.1 \%)$ & $4(30.7 \%)$ & 0.219 \\
\hline Mucocutaneous manifestation & $25(69.4 \%)$ & $6(66.6 \%)$ & $10(71.4 \%)$ & $9(69.2 \%)$ & 0.276 \\
\hline Gastrointestinal manifestation & $24(66.6 \%)$ & $4(44.4 \%)$ & $8(57.1 \%)$ & $12(92.3 \%)$ & $0.04^{\ddagger \neq}$ \\
\hline Gastroenteritis & $10(27.7 \%)$ & $2(22.2 \%)$ & $3(21.4 \%)$ & $5(38.4 \%)$ & 0.560 \\
\hline Hepatitis & $9(25 \%)$ & $1(11.1 \%)$ & $3(21.4 \%)$ & $5(38.4 \%)$ & 0.320 \\
\hline Mesenteric lymphadenopathy & $5(13.8 \%)$ & $1(11.1 \%)$ & - & $4(30.7 \%)$ & $0.011^{¥}$ \\
\hline Ascites & $4(11.1 \%)$ & - & $2(14.2 \%)$ & $2(15.3 \%)$ & 0.470 \\
\hline Neurological manifestation & $14(38.8 \%)$ & $4(44.4 \%)$ & $6(42.8 \%)$ & $4(30.7 \%)$ & 0.752 \\
\hline Headache & $3(8.3 \%)$ & - & - & $3(23 \%)$ & 0.055 \\
\hline Agitation & $7(19.4 \%)$ & $2(22.2 \%)$ & $4(28.5 \%)$ & $1(7.6 \%)$ & 0.380 \\
\hline Aseptic meningitis & $4(11.1 \%)$ & $2(22.2 \%)$ & $2(14.2 \%)$ & - & 0.197 \\
\hline Lung involvement & $3(8.3 \%)$ & - & $1(7.1 \%)$ & $2(15.3 \%)$ & 0.214 \\
\hline Parenchyma infiltration & $3(8.3 \%)$ & - & $1(7.1 \%)$ & $2(15.3 \%)$ & 0.429 \\
\hline Pleural effusion & $2(\% 5.5)$ & - & - & $2(15.3 \%)$ & 0.154 \\
\hline Haematological involvement & $34(94.4 \%)$ & $9(100 \%)$ & $12(85.7 \%)$ & $13(100 \%)$ & 0.189 \\
\hline Thrombocytopenia & $10(27.7 \%)$ & $3(33.3 \%)$ & $3(21.4 \%)$ & $4(30.7 \%)$ & 0.788 \\
\hline Lymphopenia & $12(33.3 \%)$ & - & $6(42.8 \%)$ & $6(46.1 \%)$ & $0.049^{\dagger \dagger}$ \\
\hline D-dimer abnormality & $32(88.8 \%)$ & $8(88.8 \%)$ & $11(78.5 \%)$ & $13(100 \%)$ & 0.209 \\
\hline Coagulation disorder & $10(27.7 \%)$ & $1(11.1 \%)$ & $4(28.5 \%)$ & $5(38.4 \%)$ & 0.308 \\
\hline Prerenal insufficiency & $5(13.8 \%)$ & $1(11.1 \%)$ & $1(7.1 \%)$ & $3(23 \%)$ & 0.793 \\
\hline Cardiac manifestations & $30(83.3 \%)$ & $5(88.8 \%)$ & $12(85.7 \%)$ & $13(76.9 \%)$ & $0.022^{\ddagger}$ \\
\hline Valve insufficiency & $25(69.4 \%)$ & $5(55.5 \%)$ & $9(85.7 \%)$ & $11(84.6)$ & 0.301 \\
\hline Left ventricular dysfunction & $11(30.5 \%)$ & - & $3(21.4 \%)$ & $8(61.5 \%)$ & $0.01^{\text {竬 }}$ \\
\hline Right ventricular dysfunction & $3(8.3 \%)$ & - & - & $3(23 \%)$ & 0.062 \\
\hline Ventricular dilatation with normal EF & $5(13.8 \%)$ & - & $3(21.4 \%)$ & $2(15.3 \%)$ & 0.343 \\
\hline Coronary dilatation & $4(11.1 \%)$ & & $1(7.1 \%)$ & $3(23 \%)$ & 0.199 \\
\hline Pericardial effusion & $3(8.3 \%)$ & - & $1(7.1 \%)$ & $2(15.3 \%)$ & 0.429 \\
\hline ECG abnormality & $17(47.2 \%)$ & $1(11.1 \%)$ & $7(50 \%)$ & $9(69.2 \%)$ & $0.026^{\ddagger}$ \\
\hline Sinus tachycardia & $8(22.2 \%)$ & $1(11.1 \%)$ & $4(28.5 \%)$ & $3(23 \%)$ & 0.614 \\
\hline Incomplete right bundle branch block & $5(13.8 \%)$ & $1(11.1 \%)$ & $2(14.2 \%)$ & $2(15.8 \%)$ & 0.959 \\
\hline $1^{\circ} \mathrm{AV}$ block & $4(11.1 \%)$ & - & $1(7.1 \%)$ & $3(23 \%)$ & 0.199 \\
\hline ST elevation & $1(2.7 \%)$ & - & - & $1(7.6 \%)$ & 0.446 \\
\hline Normal ECG rhythm & $19(52.7 \%)$ & $8(88.8 \%)$ & $7(50 \%)$ & $4(30.7 \%)$ & $0.026^{\ddagger}$ \\
\hline ICU & $4(11.1 \%)$ & - & - & $4(30.7 \%)$ & 0.553 \\
\hline Vasoactive drugs & $11(30.5 \%)$ & $4(44.4 \%)$ & $3(21.4 \%)$ & $4(30.7 \%)$ & 0.505 \\
\hline Mechanical ventilation & - & - & - & - & \\
\hline ECMO & - & - & - & - & \\
\hline
\end{tabular}

Categorical variables were presented as number and percentage. Age groups were compared with $X^{2}$ test ${ }^{\dagger} p<0.05 ;<5$-year-old patients versus between 5- and 10-year-old patients

${ }^{\ddagger} p<0.05 ;>10$-year-old patients versus $<5$-year-old patients

${ }^{¥} p<0.05 ;>10$-year-old patients versus between 5- and 10-year-old patients 
membrane oxygenation. All patients were treated by IVIG ( $2 \mathrm{~g} / \mathrm{kg} /$ dose), adjunctive methylprednisolone, acetylsalicylic acid (3-5 mg/kg/day), and enoxaparin. In mild cases, methylprednisolone $(2 \mathrm{mg} / \mathrm{kg} / \mathrm{day})$ was given for 5 days, then tapered in 2-3 weeks. In moderate cases, methylprednisolone was given in a $10 \mathrm{mg} / \mathrm{kg}$ single dose, then reduced to $2 \mathrm{mg} / \mathrm{kg} / \mathrm{day}$ for 7 days and tapered in 4-6 weeks. Severe cases received methylprednisolone $20-30 \mathrm{mg} / \mathrm{kg} /$ day (max. $1 \mathrm{~g}$ ) for 1-3 days, then reduced to $2 \mathrm{mg} / \mathrm{kg} /$ day for 14 days and tapered in 6-8 weeks. Only one patient who had heart failure and coronary dilatation needed a second dose of IVIG because of persistent fever after $36 \mathrm{~h}$ of IVIG treatment. Nineteen patients with ventricular dysfunction and/or coronary dilatation were treated by therapeutic-dose enoxaparin with a median duration of 14 days (range: 6-26). Treatment was discontinued in one patient due to epistaxis. The other patients received prophylactic dosages of enoxaparin with a median duration of eight days (range: 2-18). The enoxaparin treatment was continued until cardiac function recovery and the D-dimer level was reduced below $1000 \mathrm{ng} / \mathrm{mL}$.

On admission, broad-spectrum empiric antibiotic treatment was initiated in 26 patients. All blood and urine cultures were negative. All patients responded dramatically to IVIG and steroid therapy and remained fever-free. No patients needed immunomodulatory therapy. No patient died. On the 1st week control of echocardiography, all previously defined multiple valve insufficiency, pericardial effusion, right ventricular dysfunction, and ventricular dilatation were completely recovered. Left ventricular dysfunction and coronary dilatation recovered at the end of the 2nd week. Cardiac dysfunction resolved completely in all patients before discharge, except for one patient who had mild coronary artery dilation. At the time of writing of the manuscript, his control echocardiography revealed normal coronary arteries.

The median recovery days of for laboratory values are as follows: ESR, 9 (range: 3-35) days; CRP, 7 (range: 2-13) days; procalcitonin, 6 (range: 1-10) days; IL-6, 4.5 (range: 2-15) days; ferritin, 6 (range: 4-10) days; D-dimer, 7 (range: 3-16) days; Pro-BNP, 11 (range: 3-19) days; and troponin, 7 (range: 2-18) days. Patients were discharged with low-dose ASA therapy, which was stopped if the 8th week ECHO finding was normal.

\section{Discussion}

The clinical spectrum of MIS-C ranges from mild to severe multisystem involvement [6,7]. One of the initial challenges' clinicians face is being able to recognize MIS-C cases without KD features because myocarditis can evolve rapidly and needs to be identified early. In the present study, we compared the characteristics of MIS-C cases according to the severity of the disease and by age groups. Critical cardiac involvement was detected in all age groups and clinical phenotypes. We observed that MIS-C tends to affect all paediatric age groups, especially children older than 5 years.

In previous studies, there was no gender dominance in MIS-C patients [10]. The majority of patients were previously healthy, while obesity (25\%) and asthma (19\%) were the most commonly reported comorbidities [11]. In our study, except for one obese adolescent $(2.7 \%)$, the remaining patients were previously healthy. COVID-19 was confirmed in $91.6 \%$ of the patients using antibody or RT-PCR tests. Epidemiological history was positive in $55.5 \%$ of patients. Feldstein et al. indicated that $70 \%$ of patients had positive RT-PCR or antibody testing and 30\% had exposure to a person with COVID-19 within the last 4 weeks [12].

Our observations about clinical presentations of MIS-C were very similar to those previously reported in the literature [13]. A systemic review including 875 MIS-C patients of Panigrahy et al. [13] concluded that GI symptoms, including abdominal pain (52.8\%), vomiting (44.8\%), diarrhoea (39.5\%), and mucocutaneous symptoms (44.4\%), were common. Respiratory distress (20.9\%) and neurological involvement (17.5\%) were less common.

Although some clinical features are similar, MIS-C cases differ from KD in a few ways, including older age, profound form of inflammation, and prominent gastrointestinal and neurologic symptoms, with such different laboratory findings as lymphopenia, thrombocytopenia, elevated troponin, pro-BNP, D-dimer, and ferritin levels. MIS-C cases are less likely to be Caucasians and Asians $[6,14]$. While MIS-C patients often exhibit cardiac dysfunction and hypotension, coronary artery dilatation is mild and transient compared with KD [3]. In our study, MIS-C overlapping with KD was the most common clinical appearance in all age groups (69.4\%). We observed that common presenting symptoms were fever (100\%), mucocutaneous symptoms (69.4\%), including conjunctivitis and rash; gastrointestinal symptoms (66.6\%), including vomiting, abdominal pain, and/or diarrhoea; and neurological findings (38.8\%). Cardiovascular (83.3\%) and hematologic system (88.8\%) involvements were found to be especially high. Cervical lymphadenopathy, however, was less common $(13.8 \%)$ compared to KD. Respiratory symptoms overlapping with severe acute COVID-19 were less common $(n=3,8.3 \%)$, and SARS-CoV-2 PCR positivity was detected in only one of our patients. As of July 29, 2020, a total of 570 MIS-C patients were reported to the CDC in the US clinical features of $22.6 \%$ of the patients resembled features of acute COVID-19 pneumonia. The rate of SARS-CoV-2 RT-PCR positivity (84.0\%), case mortality (5.3\%), and median age of patients was higher than in other clinical spectra [15]. Dufort et al. [10] reported that MIS-C 
presentation varies by age. While younger patients more often exhibited KD features, older patients were admitted with more myocarditis. In our study, myocarditis (52.7\%) was detected in all age groups and all clinical phenotypes of MIS-C. Longer fever duration and high-grade fever were identified as a risk factor for myocarditis. Hepatitis was detected in 25\% of MIS-C cases, regardless of the severity of the disease, and resolved in all patients by 2 weeks post-discharge. On the other hand, high incidence rates of hepatitis (43\%) were reported as associated with the severity of MIS-C in Cantor et al.'s study [16]. Interestingly, co-existence of epididymo-orchitis and KD features was not previously reported. We think that clinicians should be alert to this different presentation of MIS-C.

As in most other previously reported MIS-C cohorts [4], our study detected lymphopenia (33.3\%), thrombocytopenia $(27.7 \%)$, and high inflammatory markers, such as CRP (97.2\%), ESR (75\%), procalcitonin (75\%), IL-6 (86.1\%), and ferritin (44.4\%). Lymphopenia, high levels of procalcitonin, IL-6, ferritin, D-dimer, troponin, pro-BNP, urea, and ALT were also related to the severity of MIS-C. However, acute phase reactants, biochemistry, and cardiac enzymes showed no differences related to the clinical phenotype of MIS-C.

Chest imaging features of MIS-C can mimic acute COVID-19 infection. Lima-Setta's study reported that ground-glass opacities were observed in $31 \%$ of patients [17]. Whereas in our cohort, however, pulmonary involvement, including ground-glass opacities and local patchy shadowing, was rare. Different findings may be detected in abdominal US due to MIS-C. Mamishi et al. found that the frequency of pleural effusion, ascites, ileitis, and pericardial effusions was $18 \%, 11 \%, 4 \%$, and $2 \%$ of cases, respectively [4]. We detected in our patients' occurrences of mesenteric lymphadenitis (13.8\%), pericardial effusions (8.3\%), pleural effusion (5.5\%), ileitis (2.7\%), cholecystitis (2.7\%), and ascites (1.1\%).

Although cardiovascular complications are the most prominent manifestations of MIS-C, the frequency of cardiac involvement is unclear. Minocha et al. showed that majority of patients (78\%) had at least one abnormality in cardiac testing, including abnormal ECHO (30\%), abnormal ECG (48\%), elevated pro-BNP (43\%), and/or elevated troponin (21\%) [18]. Severe MIS-C cases can present as vasodilatory or cardiogenic shock, often requiring inotropic and vasopressor support, mechanical ventilation, and extracorporeal membrane oxygenation [19]. In our study, on admission, nearly one-third of the patients presented with cardiogenic shock and $30.5 \%$ cases required inotropic support. Recent large case series reported that approximately 30 to $40 \%$ of children with MIS-C had depressed left ventricular function, 8 to $19 \%$ had coronary artery abnormalities, and 40 to $80 \%$ had myocarditis $[6,12,15,20]$. We showed that valve insufficiency $(69.4 \%)$ and left ventricular dysfunction (30.5\%) were the most common ECHO abnormalities. The most frequently reported ECG anomalies were non-specific ST segment changes, QTc prolongation, and premature atrial or ventricular beats. First- and seconddegree AV blocks were reported in one series, while atrial fibrillation was described in two reports [21, 22]. In our study, the most common ECG abnormalities on admission were sinus tachycardia (22.2\%), and during hospitalisation, sinus arrhythmia was detected in $33.3 \%$ of the patients. Non-sustained uniform ventricular tachycardia temporarily occurred in two patients with severe myocarditis resolved spontaneously. We suggest that regardless of the severity of the disease, rhythm monitoring should be performed in all MIS-C cases. In the literature, elevation of pro-BNP and troponin has been reported at different rates depending on cardiac involvement [11]. We confirmed the diagnosis of MIS-C with abnormal ECHO findings in eight patients who presented with only gastrointestinal symptoms. In all of them, pro-BNP levels were high on admission. We also recommend that the pro-BNP test be used as a screening test in children with prolonged and unexplained fever to determine early cardiac involvement of MIS-C.

Dysregulated immune response with suppression of interferon responses and concurrent hyperproduction of other cytokines (e.g., IL-6 and tumour necrosis factor (TNF)) was considered to responsible in patogenesis of MIS-C. Elevated interleukin 1 (IL-1) was also reported in MIS-C cases in the literature [23]. Although we studied only IL-6 levels, we think that other cytokines might also be responsible for MIS-C pathogenesis.

While today the optimal therapeutic strategy for MIS-C remains unknown, there are different treatment modalities. Glucocorticoid indiscriminates immunosuppressants, reduces production of proinflammatory cytokines (e.g., IL-2, IL-6, and TNF), and suppresses activation of T cells, monocytes, and macrophages. IVIG reduce antibodydependent enhancement of the immune response, and binding of proinflammatory cytokine [24]. The most common treatment strategy included empirical IVIG combined with corticosteroids, and some cases even required biological agents such as a tumour necrosis factor inhibitor or IL-1 inhibitor. Feldstein et al. reported that patients with MIS-C received IVIG (77\%), glucocorticoids (49\%), biological agent (20\%), and vasoactive support (48\%) [12]. Although steroid treatment for every patient is controversial, many studies indicated that combined treatment with methylprednisolone versus IVIG alone was associated with a better course of fever, less severe acute complications, shorter cardiac recovery, and reduced mortality in MIS-C [25, 26]. All our patients were started with IVIG $(2 \mathrm{~g} / \mathrm{kg})$ and corticosteroid dosages adjusted according to clinical severity, and they became afebrile in $48 \mathrm{~h}$. None of the patients required IL-1 receptor antagonist A-anakinra treatment. 
MIS-C cases are at risk for thrombotic complications due to endothelial injury, immobilization, ventricular dysfunction, and coronary artery aneurysm. Antiplatelet and/or anticoagulation treatment is recommended, based on coagulation tests and clinical presentation [2]. Therefore, regardless of the clinical features of MIS-C cases, low-dose ASA and prophylactic or treatment-dose enoxaparin were initiated in our patients. Cardiac functions, and pathological findings in lung and abdominal imaging, quickly recovered in the 2-week controls, except for one patient with mild coronary artery dilation. We think that a favourable outcome can be achieved with administration of IVIG and steroid therapy in the early stage of MIS-C.

Our study has some limitations. It is a single-centre and retrospective study which had a relatively small number of cases. MIS-C is recently described as a life-threatening disorder needing urgent management, and there is no proven curative therapy. We used recommended therapies based on worldwide experience of MIS-C and could not compare different treatment regimens. Therefore, prospective, multicentre, and even randomized studies are needed to confirm these observations. Additionally, lumbar puncture could not be performed in patients with suspected neurological involvement because of their unstable hemodynamic status or their disapproval of the procedure.

The diagnosis of MIS-C requires special attention due to its unique clinical manifestations with multisystemic involvement and the inability to obtain detailed epidemiological histories. As SARS-COV-2 continues to circulate around the world, MIS-C should be considered in all children with fever. In suspected MIS-C cases, early cardiac evaluation can provide early diagnosis, and favourable outcomes can be achieved with intensive immunomodulatory therapy together with high doses of IVIG and corticosteroids.

\footnotetext{
Author contributions Gulsum Alkan: Substantial contributions to conception and design, acquisition of data, analysis and interpretation of data, and drafting the article and revising it critically for important intellectual content. Ahmet Sert: Substantial contributions to conception and design, acquisition of data, analysis and interpretation of data, and drafting the article. Sadiye Kubra Tuter Oz: Substantial contributions to conception and design, acquisition of data, analysis and interpretation of data, and revising it critically for important intellectual content. Melike Emıroglu: Substantial contributions to conception and design, acquisition of data, analysis and interpretation of data, and revising it critically for important intellectual content. Resul Y1lmaz: Substantial contributions to conception and design, acquisition of data, analysis and interpretation of data, and revising it critically for important intellectual content. We further confirm that any aspect of the work covered in this manuscript that has involved either experimental animals or human patients has been conducted with the ethical approval of all relevant bodies and that such approvals are acknowledged within the manuscript.
}

Data availability The authors confirm that the data supporting the findings of this study are available within the article and/or its supplementary materials.

Code availability All source codes are available within the article and/ or its supplementary materials.

\section{Declarations}

Ethics approval The study protocols were approved by Selcuk University Faculty of Medicine ethics committee (approval number: 2021/58).

Consent to participate We confirm that the manuscript has been read and approved by all named authors and that there are no other persons who satisfied the criteria for authorship but are not listed. We further confirm that the order of authors listed in the manuscript has been approved by all of us.

Consent for publication All authors approved the final manuscript as submitted and agree to be accountable for all aspects of the work. Manuscript is not under publication or consideration for publication elsewhere.

Disclosures None.

\section{References}

1. Noval Rivas M, Porritt RA, Cheng MH et al (2021) COVID19-associated multisystem inflammatory syndrome in children (MIS-C): a novel disease that mimics toxic shock syndromethe superantigen hypothesis. J Allergy Clin Immunol 147:57-59. https://doi.org/10.1016/j.jaci.2020.10.008

2. Sperotto F, Friedman KG, Son MBF et al (2021) Cardiac manifestations in SARS-CoV-2-associated multisystem inflammatory syndrome in children: a comprehensive review and proposed clinical approach. Eur J Pediatr 180:307-322. https://doi.org/10.1007/ s00431-020-03766-6

3. Nakra NA, Blumberg DA, Herrera-Guerra A, Lakshminrusimha S (2020) Multi-system inflammatory syndrome in children (MIS-C) following SARS-CoV-2 infection: review of clinical presentation, hypothetical pathogenesis, and proposed management. Children 7:69. https://doi.org/10.3390/children7070069

4. Mamishi S, Movahedi Z, Mohammadi M et al (2020) Multisystem inflammatory syndrome associated with SARS-CoV-2 infection in 45 children: a first report from Iran. Epidemiol Infect 148. https:// doi.org/10.1017/S095026882000196X

5. Alsaied T, Tremoulet AH, Burns JC et al (2021) Review of cardiac involvement in multisystem inflammatory syndrome in children. Circulation 143:78-88. https://doi.org/10.1161/CIRCULATIO NAHA.120.049836

6. Whittaker E, Bamford A, Kenny J et al (2020) Clinical characteristics of 58 children with a pediatric inflammatory multisystem syndrome temporally associated with SARS-CoV-2. JAMA 324:259-269. https://doi.org/10.1001/jama.2020.10369

7. Rowley AH, Shulman ST, Arditi M (2020) Immune pathogenesis of COVID-19-related multisystem inflammatory syndrome in children. J Clin Invest 130:5619-5621. https://doi.org/10.1172/ JCI143840

8. Centers for Disease Control and Prevention (2020) Multisystem inflammatory syndrome in children (MIS-C) associated with coronavirus disease 2019 (COVID-19). August 14:1074-80 
9. Jonat B, Gorelik M, Boneparth A et al (2021) Multisystem inflammatory syndrome in children associated with coronavirus disease 2019 in a children's hospital in New York city: patient characteristics and an institutional protocol for evaluation, management, and follow-up. Pediatr Crit Care Med 22:e178-e191. https://doi.org/ 10.1097/PCC.0000000000002598

10. Dufort EM, Koumans EH, Chow EJ et al (2020) Multisystem inflammatory syndrome in children in New York State. N Engl J Med 383:347-358. https://doi.org/10.1056/nejmoa2021756

11. Blumfield E, Levin TL, Kurian J et al (2021) Imaging findings in multisystem inflammatory syndrome in children (MIS-C) associated with coronavirus disease (COVID-19). Am J Roentgenol 216:507-517. https://doi.org/10.2214/AJR.20.24032

12. Feldstein LR, Rose EB, Horwitz SM et al (2020) Multisystem inflammatory syndrome in U.S. children and adolescents. N Engl J Med 383:334-346. https://doi.org/10.1056/NEJMoa2021 680

13. Panigrahy N, Policarpio J, Ramanathan R (2020) Multisystem inflammatory syndrome in children and SARS-CoV-2: a scoping review. J Pediatr Rehabil Med 13:301-316. https://doi.org/ 10.3233/PRM-200794

14. Loke YH, Berul CI, Harahsheh AS (2020) Multisystem inflammatory syndrome in children: is there a linkage to Kawasaki disease? Trends Cardiovasc Med 30:389-396. https://doi.org/10.1016/j. tcm.2020.07.004

15. Godfred-Cato S, Bryant B, Leung J et al (2020) COVID-19associated multisystem inflammatory syndrome in children United States, March-July 2020. MMWR Morb Mortal Wkly Rep 69:1074-1080. https://doi.org/10.15585/mmwr.mm6932e2

16. Cantor A, Miller J, Zachariah P et al (2020) Acute hepatitis is a prominent presentation of the multisystem inflammatory syndrome in children: a single-center report. Hepatology 72:15221527. https://doi.org/10.1002/hep.31526

17. Lima-Setta F, de Magalhães-Barbosa MC, Rodrigues-Santos G et al (2020) Multisystem inflammatory syndrome in children (MIS-C) during SARS-CoV-2 pandemic in Brazil: a multicenter, prospective cohort study. J Pediatr (Rio J). https://doi.org/10. 1016/j.jped.2020.10.008

18. Minocha PK, Phoon CKL, Verma S, Singh RK (2021) Cardiac findings in pediatric patients with multisystem inflammatory syndrome in children associated with COVID-19. Clin Pediatr (Phila) 60:119-126. https://doi.org/10.1177/0009922820961771
19. Weatherhead JE, Clark E, Vogel TP et al (2020) Inflammatory syndromes associated with SARS-cov-2 infection: dysregulation of the immune response across the age spectrum. J Clin Invest 130:6194-6197. https://doi.org/10.1172/JCI145301

20. Kabeerdoss J, Pilania RK, Karkhele R et al (2021) Severe COVID19, multisystem inflammatory syndrome in children, and Kawasaki disease: immunological mechanisms, clinical manifestations and management. Rheumatol Int 41:19-32. https://doi.org/10. 1007/s00296-020-04749-4

21. Deza Leon MP, Redzepi A, McGrath E et al (2020) COVID19-associated pediatric multisystem inflammatory syndrome. J Pediatric Infect Dis Soc 9:407-408. https://doi.org/10.1093/jpids/ piaa061

22. Ramcharan T, Nolan O, Lai CY et al (2020) Paediatric inflammatory multisystem syndrome: temporally associated with SARSCoV-2 (PIMS-TS): cardiac features, management and short-term outcomes at a UK tertiary paediatric hospital. Pediatr Cardiol 41:1391-1401. https://doi.org/10.1007/s00246-020-02391-2

23. Cabler SS, French AR, Orvedahl A (2020) A cytokine circus with a viral ringleader: SARS-CoV-2-associated cytokine storm syndromes. Trends Mol Med. 26(12):1078-1085. https://doi.org/10. 1016/j.molmed.2020.09.012

24. Nissen CB, Sciascia S, de Andrade D, Atsumi T, Bruce IN, Cron RQ et al (2021) The role of antirheumatics in patients with COVID-19. Lancet Rheumatol. https://doi.org/10.1016/S26659913(21)00062-X

25. Belhadjer Z, Méot M, Bajolle F et al (2020) Acute heart failure in multisystem inflammatory syndrome in children in the context of global SARS-CoV-2 pandemic. Circulation 142:429-436. https:// doi.org/10.1161/CIRCULATIONAHA.120.0483

26. Ouldali N, Toubiana J, Antona D et al (2021) Association of intravenous immunoglobulins plus methylprednisolone vs immunoglobulins alone with course of fever in multisystem inflammatory syndrome in children. JAMA 325:855-864. https://doi.org/ 10.1001/jama.2021.0694

Publisher's note Springer Nature remains neutral with regard to jurisdictional claims in published maps and institutional affiliations. 